\title{
MONADIC PROPERTIES OF UNIFORMITIES
}

\author{
D. G. COYLE AND M. E. SZABO
}

\begin{abstract}
We introduce the nonstandard concept of a monadic set and characterize diagonal and covering uniformities in terms of such sets. This formulation relates the two fundamental aspects of uniformities directly and obviates the need for bases. We illustrate the utility of our approach by showing that it leads to significant simplifications of the standard proofs characterizing pseudo-metrizable uniform and uniformizable topological spaces.
\end{abstract}

1. Monadic sets. We presuppose familiarity with the basic ideas of nonstandard analysis. For notations and definitions we refer to [5] and for the general theory of monads to [2 and 3]. We also assume as given a sufficiently saturated nonstandard extension of the standard universe of uniform spaces.

Definition 1.1. A subset $M$ of a set ${ }^{*} X$ is monadic in a family $\mathfrak{B}$ of internal subsets of ${ }^{*} X$ if $M=\cap\{B \in \mathfrak{B} \mid M \subset B\}$.

Monadic sets generalize the monads of [2], where it is required that the internal sets $B$ are of the form ${ }^{*} S$ for standard sets $S$. Thus the monadic sets in 1.4, for example, are not usually monads in the sense of [2].

EXAMPLE 1.2. If $E$ is the set of entourages of a uniform space $X$ and $M=$ $\cap\left\{{ }^{*} B \mid B \in \mathbb{E}\right\}$, then $M$ is monadic in the family $B$ consisting of all sets ${ }^{*} B$ such that $B \subset X$ and $M \subset{ }^{*} B$.

EXAMPLE 1.3. If $\mathfrak{D}_{x}$ is the neighbourhood system of a point $x$ of a topological space $X$ and $M=\cap\left\{{ }^{*} G \mid G \in \mathfrak{D}_{x}\right\}$, then $M$ is monadic in the family $\mathfrak{B}$ consisting of all sets ${ }^{*} G$ such that $G \in \mathcal{D}_{x}$ and $M \subset{ }^{*} G$.

EXAMPLE 1.4. If $P={ }^{*} S$ is a partition of ${ }^{*} X, P[x] \in P$ the equivalence class of $x$, and $\operatorname{Cov}_{P}(X)$ the set of covers of $X$ with the property that for each $C \in \operatorname{Cov}_{P}(X), P$ refines ${ }^{*} C$, then $P[x]$ is monadic in the family $\mathfrak{B}$ consisting of all $\operatorname{St}\left(x,{ }^{*} C\right)$, with $C \in \operatorname{Cov}_{P}(X)$.

Examples 1.3 and 1.4 motivate the following two definitions:

Definition 1.5. An equivalence relation $\approx$ is monadic in $*(X \times X)$ if $\approx$ is monadic in the family $B$ consisting of all sets ${ }^{*} B$ such that $B \subset X \times X$ and $\approx \subset{ }^{*} B$.

Definition 1.6. A partition $\pi$ of ${ }^{*} X$ is monadic if each $\pi[x]$ is monadic in the sense of 1.4 .

Received by the editors August 20, 1982

1980 Mathematics Subject Classification. Primary 54E15, 54E25, 54J05; Secondary 03 H05.

Key words and phrases. Monadic sets, uniformities, pseudo-metrics, nonstandard, pseudo-metrizable, uniformizable, topological.

${ }^{1}$ The research of the second author is supported by the Natural Sciences and Engineering Research Council of Canada and by the Fonds F.C.A.C. of the Province of Quebec. 
We now show that monadic equivalence relations characterize diagonal uniformities, and monadic partitions characterize covering uniformities.

THEOREM 1.7. An equivalence relation $\approx$ is monadic in $*(X \times X)$ if and only if the set $\mathfrak{B}=\left\{B \subset X \times X \mid \approx \subset^{*} B\right\}$ is a diagonal uniformity on $X$.

Proof. Necessity. Suppose that $\approx=\cap\left\{{ }^{*} B \mid B \subset X \times X\right.$ and $\left.\approx \subset{ }^{*} B\right\}$ and let $B \in \mathfrak{B}$. Then $(x, x) \in{ }^{*} B$ for all $x \in{ }^{*} X$. By Transfer we therefore have $\Delta \subset B$. Now if $B_{1}, \ldots, B_{n} \in \mathfrak{B}$, then $B=B_{1} \cap \cdots \cap B_{n} \in \mathfrak{B}$, i.e., we have for any $B_{1}, \ldots, B_{n}$ $\in \mathfrak{B}$ an element $B \in \mathfrak{B}$ such that $B \subset B_{1}, \ldots, B \subset B_{n}$. By Saturation there therefore exists a $D \in * \mathscr{B}$ such that $D \subset{ }^{*} B$ for all $B \in \mathfrak{B}$. Thus $D \subset \approx$. By the symmetry and transitivity of $\approx$ we have $D \circ D \subset \approx$ and $D^{-1} \subset \approx$. Hence for any $B \in \mathfrak{B}$ there exists a $D \in *^{*} \mathscr{B}$ such that $D \circ D \subset{ }^{*} B$ and $D^{-1} \subset{ }^{*} B$. By Transfer we have a $D \in \mathfrak{B}$ such that $D \circ D \subset B$ and $D^{-1} \subset B$ for all $B \in \mathfrak{B}$. The remaining clauses hold trivially.

Sufficiency. Let $\mathfrak{B}$ be a diagonal uniformity on $X$ and let $\approx$ be defined by $\cap\left\{{ }^{*} B \mid B \in \mathfrak{B}\right\}$. It suffices to show that $\approx$ is an equivalence relation. Since $\Delta \subset B$ for all $B$, we have ${ }^{*} \Delta \subset{ }^{*} B$. But ${ }^{*} \Delta$ is clearly the diagonal of ${ }^{*} X$. Now suppose that $(x, y)$ and $(y, z)$ belong to $\approx$. Then $(x, y)$ and $(y, z)$ belong to ${ }^{*} B$ for all $B \in \mathfrak{B}$. Let $B, B^{\prime} \in \mathfrak{B}$ be such that $B \circ B \subset B^{\prime}$. Then it follows that ${ }^{*} B \circ{ }^{*} B \subset{ }^{*}\left(B^{\prime}\right)$ and therefore $(x, z) \in^{*}\left(B^{\prime}\right)$. This holds for all $B^{\prime}$. Hence $\approx$ is transitive. A similar argument establishes the symmetry of $\approx$.

We now turn to covering uniformities. We recall that a covering uniformity Unif $(X)$ on a set $X$ is a nonempty family of covers satisfying two conditions: (U1) If $C \in \operatorname{Unif}(X)$ and $C$ refines $C^{\prime}$ (written as $C<C^{\prime}$ ), then $C^{\prime} \in \operatorname{Unif}(X)$. (U2) If $C, C^{\prime} \in \operatorname{Unif}(X)$, then there exists a $C^{\prime \prime} \in \operatorname{Unif}(X)$ which star-refines both $C$ and $C^{\prime}$ (written as $C^{\prime \prime}<_{*} C$ and $C^{\prime \prime}<_{*} C^{\prime}$ ). Covering uniformities are characterized by the following two theorems:

THEOREM 1.8. For any monadic partition $\pi$ of ${ }^{*} X, \operatorname{Cov}_{\pi}(X)$ is a covering uniformity.

Proof. Condition (U1) holds trivially and it remains to prove (U2). Let $S=\left\{C_{i} \mid\right.$ $1 \leqslant i \leqslant n\}$ be a finite subset of $\operatorname{Cov}_{\pi}(X)$ and form the set $\bar{S}=\left\{\cap_{i} a_{i} \mid a_{i} \in C_{i}\right.$ and $1 \leqslant i \leqslant n\}$. Then $\bar{S}<C_{i}$ and $\pi<^{*}(\bar{S})$. Hence $\bar{S} \in \operatorname{Cov}_{\pi}(X)$. By Saturation there therefore exists a $D \in{ }^{*} \operatorname{Cov}_{\pi}(X)$ such that $D<{ }^{*} C$ for all $C \in \operatorname{Cov}_{\pi}(X)$. We claim that $D<\pi$. Let $a \in D$ and $x \in a$. For each $C \in \operatorname{Cov}_{\pi}(X)$, choose $a(C) \in{ }^{*} C$ such that $a \subset a(C)$. Then

$$
a \subset \cap\left\{a(C) \mid C \in \operatorname{Cov}_{\pi}(X)\right\} \subset \cap\left\{\operatorname{St}\left(x,{ }^{*} C\right) \mid C \in \operatorname{Cov}_{\pi}(X)\right\}=\pi[x] .
$$

But since $\pi$ is a partition, we have $\pi<{ }_{*}^{*} C$ for all $C \in \operatorname{Cov}_{\pi}(X)$, and therefore $D<\pi<{ }^{*} C$, so that $D<{ }^{*} C$ for all ${ }^{*} C \in{ }^{*} \operatorname{Cov}_{\pi}(X)$. Let $C, C^{\prime} \in \operatorname{Cov}_{\pi}(X)$. It then holds that there exists a $D$ in ${ }^{*} \operatorname{Cov}_{\pi}(X)$ with the property that $D<{ }^{*} C$ and $D<{ }_{*}^{*}\left(C^{\prime}\right)$. By Transfer there therefore exists a $D$ in $\operatorname{Cov}_{\pi}(X)$ such that $D<_{*} C$ and $D<{ }_{*} C^{\prime}$ for all $C \in \operatorname{Cov}_{\pi}(X)$.

In the other direction, we have the following result:

THEOREM 1.9. For any covering uniformity $\operatorname{Unif}(X)$ on $X$ there exists a canonical monadic partition $\pi$ of $X$ with the property that $\operatorname{Unif}(X)=\operatorname{Cov}_{\pi}(X)$. 
Proof. The partition $\pi$ defined by $\pi[x]=\cap\left\{\operatorname{St}\left(x,{ }^{*} C\right) \mid C \in \operatorname{Unif}(X)\right\}$ has the required property.

We now have a direct link between diagonal uniformities and covering uniformities which avoids the passage via bases:

THEOREM 1.10. Let $\approx$ be an equivalence relation on $* X$ and $\pi \approx$ its associated partition. Then $\approx$ is monadic if and only if $\pi \approx$ is monadic.

Proof. Necessity. For each relation $R$ with the property that $\approx \subset^{*} R$ we define a cover of $X$ by putting $R[x]=\{y \mid(x, y) \in R\}$ and letting $C_{R}=\{R[x] \mid x \in X\}$. Then $\pi[x]=\bigcap\left\{{ }^{*} R[x] \mid \approx C^{*} R\right\}$ since $\approx$ is monadic. By the standard properties of entourages we have for each such $R$ an $S$ such that $S \circ S^{-1} \subset R$. Hence $\operatorname{St}\left(x, C_{S}\right) \subset$ $R[x]$. Thus $x \in S[y]$ if and only if $(y, x) \in S$ if and only if $(x, y) \in S \subset R$. Therefore $\pi[x]=\cap\left\{\operatorname{St}\left(x,{ }^{*} C_{S}\right) \mid \approx \subset{ }^{*} S\right\}$.

Sufficiency. If $\pi_{\approx}$ is monadic, then we put $R_{C}=\cup\{a \times a \mid a \in C\}$ for each standard uniform cover $C$ of $X$. It follows that $\approx \subset^{*}\left(R_{C}\right)$ for every $C$. Now $(x, y) \in{ }^{*}\left(R_{C}\right)$ if and only if $x, y \in a$ for some $a \in{ }^{*} C$ if and only if $x \in \operatorname{St}\left(y,{ }^{*} C\right)$. Suppose that $x \approx y$. Then for some $C, x \notin \operatorname{St}\left(y,{ }^{*} C\right)$ since $\pi \approx$ is monadic. Hence $(x, y) \notin^{*}\left(R_{C}\right)$. Thus

$$
\approx=\cap\left\{{ }^{*}\left(R_{C}\right) \mid C \in \operatorname{Unif}(X)\right\}=\cap\left\{{ }^{*} R \mid \approx \subset{ }^{*} R \text { and } R \subset X \times X\right\} .
$$

2. Pseudo-metrizable uniform spaces. In the light of $\S 1$, we can think of a uniform space as a pair $(X, \approx)$ with $\approx$ a monadic equivalence relation on $*(X \times X)$. We say that a cover $\mathfrak{U}$ of $X$ is uniform with respect to $\approx$ if $\pi_{\approx}<* \mathfrak{U}$, and call a pseudometric $\rho$ compatible with $\approx$ if every cover of $X$ by $\rho$-balls of a fixed radius is uniform with respect to $\approx$. Our applications of the nonstandard theory are based on the following nonstandard version of a well-known result:

LeMma 2.1. For every uniform cover $\mathfrak{U}$ of a uniform space $(X, \approx)$ there exists a compatible pseudo-metric $\rho$ so that the cover of $X$ by $\rho$-unit balls refines $\mathfrak{U}$.

Proof. Let $\left(\mathfrak{U}_{i} \mid i \in \mathbf{N}\right)$ be a sequence of uniform covers of $X$ for which $\mathfrak{U}_{1}=\mathfrak{U}$ and $\mathfrak{U}_{i+1}<{ }_{*} \mathfrak{U}_{i}$. Then ${ }^{*}\left(\mathfrak{U}_{i} \mid i \in \mathbf{N}\right)=\left(\mathfrak{B}_{i} \mid i \in{ }^{*} \mathbf{N}\right)$, where $\mathfrak{B}_{i}={ }^{*} \mathfrak{U}_{i}$ for each $i \in \mathbf{N}$. Let $n \in{ }^{*} \mathbf{N}-\mathbf{N}$. Then $\left(\mathfrak{B}_{1}, \ldots, \mathfrak{B}_{n}\right)$ is a hyperfinite sequence of covers of ${ }^{*} X$ with the property that $\mathfrak{B}_{1}={ }^{*} \mathfrak{U}_{1}={ }^{*} \mathfrak{U}$ and $\mathfrak{B}_{i+1}<* \mathfrak{B}_{i}$. Define $t:{ }^{*} X \times{ }^{*} X \rightarrow{ }^{*} \mathbf{R}$ as follows:

$$
t(x, y)= \begin{cases}0 & \text { if } \operatorname{St}\left(x, \mathfrak{B}_{n}\right)=\operatorname{St}\left(y, \mathfrak{B}_{n}\right), \\ 2^{-n} & y \in \operatorname{St}\left(x, \mathfrak{B}_{n}\right), \\ 2^{-i} & y \in \operatorname{St}\left(x, \mathfrak{B}_{i}\right), y \notin \operatorname{St}\left(x, \mathfrak{B}_{i+1}\right), \\ 1 & y \notin \operatorname{St}\left(x, \mathfrak{B}_{1}\right) .\end{cases}
$$

We can show that $t(x, x)=0$ and that $t(x, y)=t(y, x)$. Moreover, for any four points $x, y, w, z \in{ }^{*} X$ it follows from the defining properties of star refinements that $t(x, y) \leqslant 4 \cdot \max (t(x, z), t(z, w), t(w, y))$.

Among all hyperfinite paths $\left(x_{i}\right)$ from $x$ to $y$ in ${ }^{*} X$ there are some for which $\Sigma_{i} t\left(x_{i-1}, x_{i}\right)$ is a minimum, and among these there are some of minimum length. 
Call such paths minimal and define $d(x, y)=\Sigma_{i} t\left(x_{i-1}, x_{i}\right)$ along a minimal path from $x$ to $y$. By induction on path length we conclude that for any minimal path $\left(x_{i} \mid 0 \leqslant i \leqslant m \in{ }^{*} \mathbf{N}\right)$ there exists a unique $j$ between 0 and $m$ such that $t\left(x_{0}, x_{j-1}\right)$, $t\left(x_{j}, x_{m}\right) \leqslant t\left(x_{j-1}, x_{j}\right)=\max _{i} t\left(x_{i-1}, x_{i}\right)$. As an immediate consequence we have that for any $x, y \in{ }^{*} X, t(x, y) \leqslant 2^{-n}$ implies that $d(x, y) \leqslant 2^{-n}$. For $x, y \in X$ we can thus put $\rho(x, y)=$ the standard part of $(4 \cdot d(x, y))$. The function $\rho$ is a pseudo-metric and the cover $\mathfrak{U}$ is refined by unit balls since $\rho(x, y)<1$ implies that $d(x, y) \leqslant 2^{-2}$, and therefore $t(x, y) \leqslant 2^{-2}$, i.e., $y \in \operatorname{St}\left(x, \mathfrak{U}_{2}\right) \subset a$ for some $a \in \mathfrak{H}$. To show the compatibility of $\rho$ with $\approx$ we let $r>0$ and choose $k$ so that $4 \cdot\left(2^{-k}\right)<r$. Then $\mathfrak{U}_{k}$ refines the cover consisting of balls of radius $r$. Let $y \in \mathfrak{U}_{k}$ and fix $x \in X$. Then $y \in a$ implies $\rho(x, y) \leqslant 2^{2-k}<r$. Hence $y$ belongs to the $\rho$-ball of radius $r$ centred at $x$.

As an easy corollary of Lemma 2.1, we obtain

TheOREM 2.2 (AlEXANDROFF-URYSOHN). A uniform space is pseudo-metrizable if and only if it has a countable base.

In nonstandard terms, a uniform space $(X, \approx)$ is pseudo-metrizable if there exists a pseudo-metric $\rho$ on $X$ such that $x \approx y$ if and only if ${ }^{*} \rho(x, y)$ is infinitesimal. Moreover, a set $\left\{\mathfrak{U}_{n} \mid n \in \mathbf{N}\right\}$ of covers of $X$ is a countable base for $\approx$ if $\pi_{\approx}[x]=$ $\cap\left\{\operatorname{St}\left(x,{ }^{*} \mathfrak{U}_{n}\right) \mid n \in \mathbf{N}\right\}$.

ProOF OF TheOrem 2.2. The necessity of the condition is immediate.

Sufficiency. Let $\left(\mathfrak{U}_{n} \mid n \in \mathbf{N}\right)$ be a sequence of covers which forms a base for $\approx$. We may clearly assume that $\mathfrak{U}_{n+1}<_{*} \mathfrak{U}_{n}$ for each $n \in \mathbf{N}$. Let $\rho$ be defined as in Lemma 2.1 , and let $\approx \rho$ be the uniformity induced by $\rho$, i.e., $x \approx \rho y$ if and only if ${ }^{*} \rho(x, y)$ is infinitesimal. Then $\approx \subset \approx{ }_{\rho}$. It therefore remains to show that $\approx_{\rho} \subset \approx$. Since $\rho(x, y)<2^{2-n}$ implies that $y \in \operatorname{St}\left(x, \mathfrak{U}_{n}\right)$, we know that if $y \approx_{\rho} x$, then $y \in \operatorname{St}\left(x,{ }^{*} \mathfrak{U}_{n}\right)$ for all $n \in \mathbf{N}$ and, hence, $y \approx x$.

3. Uniformizable topological spaces. We recall that in nonstandard terms a topology on a set $X$ is given by a map $\tau: X \rightarrow 2^{\left({ }^{*} X\right)}$ with the property that for all $x \in X, x \in \tau(x)$ and $\tau(x)=\cap\left\{{ }^{*} B \mid B \subset X\right.$ and $\left.\tau(x) \subset{ }^{*} B\right\}$. For a given uniformity $\approx$ on $X$, we put $\tau_{\approx}(x)=\left\{y \in \in^{*} X \mid y \approx x\right\}$. In this formulation a topological space $(X, \tau)$ is uniformizable if $\tau(x)=\tau_{\approx}(x)$ for some uniformity $\approx$ on $X$ and all $x \in X$.

The following well-known theorem is a further corollary of Lemma 2.1:

TheOrem 3.1 (WeIL). A topological space is uniformizable if and only if it is completely regular.

Proof. Necessity. Suppose that $\tau=\tau \approx$ for some $\approx$ and let $C$ be a closed subset of $X$ with $x \notin C$. It follows from these assumptions that there exists a uniform cover $\mathfrak{U}$ with the property that $\operatorname{St}(x, \mathfrak{U}) \cap C=\varnothing$. Define $\rho$ as in Lemma 2.1 and let $f$ : $X \rightarrow[0,1]$ be the function given by $f(y)=\rho(y, C)$. Then $f$ is clearly continuous and an inspection of the defining conditions of the function $t$ underlying $\rho$ shows that $f(C)=0$ and $f(x)=1$.

Sufficiency. Let $S$ be the set of continuous real-valued functions on $X$, i.e., $f \in S$ if $y \in \tau(x)$ implies $f(y) \in \tau(f(x))$ for all $x \in X$. For each $f \in S$ and positive real 
number $\varepsilon$, define $D(f, \varepsilon)=\{(x, y) \in X \times X|| f(x)-f(y) \mid<\varepsilon\}$, and put $\approx=$ $\cap\left\{{ }^{*} D(f, \varepsilon) \mid f \in S\right.$ and $\varepsilon$ is a positive real $\}$. Then it is easy to see that $\approx$ is a uniformity and it remains to show that $\tau=\tau \approx$. By the continuity of the functions $f$ we have that $\tau(x) \subset \tau_{\approx}(x)$ for all $x \in X$. To prove that $\tau_{\approx}(x) \subset \tau(x)$, we argue by contraposition. Suppose $y \notin \tau(x)$. Then there exists an open set $U$ such that $x \in U$ and $y \in{ }^{*}(X-U)$. By complete regularity we can therefore find a function $f \in S$ such that $f(X-U)=1$ and $f(x)=0$. It follows that $y \notin \tau \approx(x)$.

\section{REFERENCES}

1. P. Alexandroff and P. Urysohn, Une condition nécessaire et suffisante qu' une classe $(L)$ soit une classe (D), C. R. Acad. Sci. Paris 177 (1923), 1274-1277.

2. W. A. J. Luxemburg, A general theory of monads, Applications of Model Theory to Algebra, Analysis, and Probability (W. A. J. Luxemburg, ed.), Holt, Rinehart and Winston, New York, 1969, pp. 18-86.

3. M. M. Richter, Monaden, ideale Punkte und Nichtstandard-Methoden, Vieweg, Darmstadt, 1982.

4. A. Robinson, Non-standard analysis, North-Holland, Amsterdam, 1966.

5. K. D. Stroyan and W. A. J. Luxemburg, Introduction to the theory of infinitesimals, Academic Press, New York, 1976.

6. A. Weil, Sur les espaces à structure uniforme et sur la topologie générale, Actualités Sci. Indust., No. 551, Hermann, Paris, 1937.

Department of Mathematics, Concordia University, Montreal, Quebec, Canada H3G 1 M8 